\title{
Editorial
}

\section{Indian Women at high Risk for Vascular Disease}

\author{
Paturi Vishnupriya Rao ${ }^{1}$ \\ ${ }^{1}$ Kumudini Devi Diabetes Research Center, Ramdevrao Hospital, \\ Hyderabad, Telangana, India \\ Ind J Car Dis Wom 2019;4:175-176
}

In this issue of the Indian Journal of Cardiovascular Diseases in Women, an original article reports the increasing burden of vascular disease in women with diabetes. The authors have recorded presenting symptoms for cardiac and other diabetic vascular diseases in women with and without diabetes, to study gender susceptibility for symptomatic heart disease. Women older than 18 years, with known hypertension, with or without diabetes, attending the endocrinology outpatient clinic of a tertiary hospital, during a 6-month period were consecutively included in this study.

About half of the study subjects had diabetes, and others were without diabetes. The authors interviewed and recorded symptoms suggesting neuropathy, coronary heart disease, nephropathy, and retinopathy, and later confirmed the presence or absence of micro- and macrovascular diseases, as part of the routine hospital practice protocol.

The gender-specific information on age, anthropometric measurements, known duration of diabetes and hypertension, gynecological history, systolic and diastolic blood pressure, and heart rate were available for analysis. Their laboratory test reports-hemoglobin and white blood cell count, blood urea; creatinine and glycemic indices such as glycated hemoglobin and blood glucose in fasting and after food; lipid levels like total cholesterol, LDLc, VLDLc, HDLc, and triglycerides; and vitamin D-were included in the study.

The metabolic risk factors stated earlier in these hypertensive women were almost similar between those with and without diabetes. However, women with diabetes had apparently much larger vascular burden. It was obvious that diabetes alone was the major risk correlate for vascular disease, among other risk variables.

In women with diabetes, nonspecific clinical presentation like chest pain, shortness of breath, palpitation, and diaphoresis evident in younger age, may not always warrant suspicion of vascular disease, often delaying diagnosis. This was evident in the present study, as about half (49\%) of women with diabetes presented with shortness of breath and fatigability followed by about one-fourth (24\%) with more specific symptomatology like chest pain, palpitations, and

Address for correspondence

Paturi Vishnupriya Rao, MD, PhD,

Kumudini Devi Diabetes Research

Center, Ramdevrao Hospital,

Kukatpally, Hyderabad 500082,

Telangana, India

(e-mail: diabetes@diabaid.in, raopaturi@gmail.com). diaphoresis, indicating probable cardiac disease. However, two-thirds (64\%) of women with or without any of these symptoms had diabetes-related cardiac disease. Among other associated complications reported in the study, peripheral neuropathy was most common in about half (47\%), followed by nephropathy and retinopathy.

These findings prompted authors to conclude that asymptomatic and symptomatic cardiac disease was more frequently seen in women with diabetes than other complications. They recommend detailed evaluation for coronary disease, neuropathy, nephropathy, and retinopathy in all women with diabetes, during their first visit itself in outpatient clinics.

The original article titled "Correlation of Incidence of Microvascular and Macrovascular Complications in Indian Diabetic versus Nondiabetic Females,"1 published in this issue of the Journal exemplifies, an important population-specific characteristic that Asian Indian women are ethnically more susceptible for developing diabetes, whether living in India or after migration into far-off countries. Such gender-specific differences were earlier occasionally reported among Indians living in South Africa, Surinam, and Fiji. In 1991, an international diabetes study of native, recently migrated, first- and second-generation Asian Indians reported male predominance (1:0.7) in rural India, equal genders (1:1) in urban India, and marked female excess (1:2.3) in Guyana. ${ }^{2}$ Now similar trends are increasingly evident from diabetes prevalence studies within India and from other countries too, ${ }^{3}$ indicating that Indian women are at higher risk for diabetes. However, except for increasing body weight, none of the known and putative diabetes risk factors seemed to have any significant causative role to adequately explain this gender effect.

Another notable feature in women was higher prevalence of the metabolic syndrome (MetS) as compared with men in both urban and rural areas, an observation reported from many regions of India. ${ }^{4}$ In rural western India, women who gained more weight during and after pregnancy were sedentary and had thicker skinfolds and showed more tendency for hyperglycemia after pregnancy.
C2019 Women in Cardiology and Related Sciences
License terms

$($ () (1) $\Theta \circledast$
DOI https://doi.org/ 10.1055/s-0039-3402691 
Apart from diabetes and MetS, prediabetes is also more common in young, adolescent, and adult females. In Telangana state, ICMR-India Diabetes (INDIAB) Study-Phase II was conducted between June 2012 and December 2013. ${ }^{3}$ Age-specific prevalence (\%) of diabetes above 25 years in urban Telangana was 14.7 in men and 9.79 in women and in rural areas of the state it was 8.31 in men and 3.94 in women. Prevalence of prediabetes in both genders was high with female excess in Telangana state, as it was 8.82 in men and 9.06 in women in urban areas, and 8.56 in men and 8.82 in women in rural areas. Similarly, female predominance among prediabetics, is also being reported from European countries. A large observational, population-based cohort study of 11,014 individuals in Austria was presented at the recently held European Association for Study of Diabetes in 2019. ${ }^{5}$ Age-specific prevalence of prediabetes between 6 and 10 years was more in girls, $4.8 \%$, versus $4.4 \%$ in boys, and $42.3 \%$ of women had prediabetes versus $40.4 \%$ of men older than 70 years, in Austria. It is likely that if women in India with prediabetes, MetS, or any grade of hyperglycemia continue with the current imbalanced lifestyle, conversion to diabetes may occur rapidly, and thus prevalence of diabetes may also rise further in women.

Diabetes in women is also known to predispose to diabetes in populations across generations. Infants of mothers with diabetes, at least in high-risk populations such as Asian Indians, are at high risk of developing diabetes as children and young adults. By the time they become pregnant, these female offspring may already have diabetes. The fetal origins of adult diseases, thus, perpetuate this vicious cycle of transgenerational inheritance of diabetes risk in Asian Indian women. ${ }^{6}$

Morbidity and mortality attributed to diabetes was distinctly different among Asian Indians compared with other populations. Between 1977 and 1987, death records of 359 men and 191 women at the All India Institute of Medical Sciences in New Delhi were analyzed for diabetes-related vascular complications. In this study, cerebrovascular disease was more often noted in women (25.13\%) than in men (22.01\%) [personal communication]. In 1993 to 1994, vascular complications at death were recorded in 292 men and 161 women in Nizam's Institute of Medical Sciences, Hyderabad. In this study, it was reported that women with diabetes and infections died earlier (age $54.6 \pm 10.6$ years) than men (age $59.3 \pm 11.54$ years, $p<0.05$ ). Reduction in life expectancy in these women was more pronounced ( $22.0 \pm 9.31$ years) as compared with men $(17.4 \pm 8.4$ years, $p<0.01)$ [personal communication].

Recently, the Vanderbilt-led research team pooled 22 prospective cohort studies in multiple countries from mainland China to India and Bangladesh. ${ }^{7}$ More than one million individuals were followed for an average of 12.6 years. Diabetes was associated with a nearly two-fold increase in the risk of death from all causes. The researchers found that women with diabetes are at a substantially elevated risk of premature death.

ICMR multicentric study conducted between 1984 and 1987 in nine teaching hospitals of India (men 2,783, women 1,854 ), aged 25 years or above at the time of first diagnosis of diabetes and were not normally receiving insulin, revealed that of all vascular diseases cerebrovascular disease or stroke was noted in $1.8 \%$ of women and $1.7 \%$ of men [personal communication]. These multicentric vascular morbidity data are in consonance with the mortality data as presented earlier. The gender differences reported in Indian studies warranted early identification of diabetic women at high risk for developing infections and their aggressive management.

In Austria, comorbidity profile associated with biological sex and behavioral gender was markedly different between male and female participants, particularly in those with prediabetes-women had more often suffered from arrhythmias, noncoronary artery disease, osteoporosis, increased systemic inflammatory biomarkers, and depression, while men more often had angina pectoris, myocardial infarction, and media sclerosis. ${ }^{5}$

Authors of the article "Correlation of Incidence of Microvascular and Macrovascular Complications in Indian Diabetic versus Nondiabetic females," published in the current issue of the Journal signify definitive risk evidence for diabetes and related morbidity in Indian women. The emerging scientific evidence for female preponderance for diabetes underscores the need for the onset of prevention of diabetes in girls at a very young age as well in women across all ages in India.

\section{Conflict of Interest}

None.

\section{References}

1 Present article going to publish in the present issue "Correlation of incidence of microvascular and macrovascular complications in Indian diabetic versus nondiabetic females" Sudhanshu et al.

2 Rao PV, Ahuja MM, Trivedi BB, et al. Age: the most significant risk for diabetes in Indian populations. J Indian Med Assoc 1998;96(5):155-157

3 Anjana RM, Deepa M, Pradeepa R, et al. ICMR-INDIAB Collaborative Study Group. Prevalence of diabetes and prediabetes in 15 states of India: results from the ICMR-INDIAB population-based cross-sectional study. Lancet Diabetes Endocrinol 2017;5(8):585-596

4 Misra R, Misra A, Kamalamma N, et al. Difference in prevalence of diabetes, obesity, metabolic syndrome and associated cardiovascular risk factors in a rural area of Tamil Nadu and an urban area of Delhi. Int J Diabetes Dev Ctries 2011;31(2):82-89

5 OfenheimerA, Breyer-KohansalR, AltzieblerJV, et al. . Differences in prediabetes and diabetes associated co-morbidities between men and women. Abstract 295. Presented at: European Association for the Study of Diabetes Annual Meeting; Sept. 16-20, 2019; Barcelona, Spain. https://www.easd.org/ virtualmeeting/home.html\#! resources/differences-in-prediabetes-and-diabetes-associated-co-morbidities-between-men-and-women-c3835847-3c05-462e-bcfd-fb83212 e502f . Accessed October 29, 2019

6 Golden SH, Yajnik C, Phatak S, Hanson RL, Knowler WC. Racial/ ethnic differences in the burden of type 2 diabetes over the life course: a focus on the USA and India. Diabetologia 2019;62(10):1751-1760

7 Yang JJ, Yu D, Wen W, et al. Association of diabetes with allcause and cause-specific mortality in asia: a pooled analysis of more than 1 million participants. JAMA Netw Open 2019;2(4):e192696 